\title{
Influence des propriétés surfaciques et volumiques des bandes flexibles lors de leur déroulement-enroulement
}

\author{
Dominique Knittel ${ }^{1,2, a}$, Vincent Gassmann ${ }^{1,3}$, David Kuhm ${ }^{1,3}$ et Marie-Ange Bueno ${ }^{3}$ \\ 1 ERT enroulement et transport à haute vitesse de bandes flexibles (ERT 8), Université Louis Pasteur, IPST, \\ 17 rue du Maréchal Lefèbvre, 67100 Strasbourg, France \\ 2 Laboratoire de Génie de la Conception (EA 3938), INSA Strasbourg, 24 boulevard de la Victoire, \\ 67084 Strasbourg Cedex, France \\ 3 Laboratoire de Physique et Mécanique Textiles (UMR 7189 CNRS/UHA), ENSISA, Université de Haute Alsace, \\ 11 rue Alfred Werner, 68093 Mulhouse Cedex, France
}

Reçu le 28 août 2008, accepté le 9 avril 2009

Résumé - La manipulation et le traitement de matériaux tels que les textiles, le papier ou le métal s'opèrent très fréquemment sous forme de rouleaux sur des systèmes de déroulement-enroulement et transport de bandes. La qualité du produit final dépend essentiellement de la capacité du système commandé à assurer un bon suivi de consigne de la vitesse et de la tension de bande, cette tâche étant rendue ardue en raison du fort couplage entre ces deux grandeurs. Par ailleurs, le changement de bobines est effectué généralement sans arrêter le procédé de fabrication. Un accumulateur, chargé de stocker au préalable la bande et de la restituer lors du changement de la bobine, est souvent utilisé. L'influence du comportement mécanique de la bande sera illustrée. Les propriétés volumiques de la bande étant fondamentales pour la commande du procédé, elles doivent être connues au préalable ou mesurées en ligne. Pour ce faire plusieurs solutions sont possibles. Une solution consiste à ajouter un capteur adéquat dont les informations servent de données d'entrée au système de régulation. Une autre approche consiste à inclure un élément mobile dans le système de déroulement-enroulement, le danseur, afin de stabiliser la tension de bande.

Mots clés : Systèmes d'enroulement-déroulement / régulation de la tension / propriétés volumiques / danseur / accumulateur / élongation de bande

\begin{abstract}
Influence of the mechanical properties of flexible webs during their unwindingrewinding. The handling of materials such as textiles, paper or metal is often processed with webs on rollers with unwinding-winding systems. The quality of the final product depends mainly on the ability of the system to control web tension and speed. This issue is difficult because of the high coupling between these two variables. Moreover, unwound roll changes are usually carried out while the process continues at a constant velocity. Accumulators which are moving mechanisms are generally used to store and bring back the web during this operation. The influence of the web mechanical behavior will be pointed in this paper. As the elastic properties are essential to achieve good control performances, they have to be known a priori or determined online. Some solutions are available. One of them is the addiction of a sensor whose data can be used as an input of the controller. Another approach deals with the use of dancer mechanisms for web tension regulation.
\end{abstract}

Key words: Web handling systems / tension control / elastic properties / dancer / accumulator / web strain

\section{Introduction}

Tout produit en feuille - tissu, papier, nappe, film polymère, métal etc. - a subi au cours de son élaboration des cycles de déroulement/traitement/enroulement. Le savoir-faire des professionnels en entraînement de bandes est la clé de la maîtrise de la production. Cette approche, acceptable pour de faibles cadences de production,

\footnotetext{
a Auteur pour correspondance :

dominique.knittel@ipst-ulp.u-strasbg.fr
}

n'est plus satisfaisante à vitesse élevée. Les contraintes actuelles de production telles que l'augmentation de la vitesse de défilement associée à un réglage précis de la tension de bande pour chaque brin, l'absence de connaissances académiques sur le comportement dynamique des systèmes d'entraînement de bandes et sur les stratégies de commande, placent les industriels dans des situations difficiles. Ils ont besoin de connaître les lois de réglage des machines pour éviter de générer des perturbations qui peuvent conduire à l'altération ou même à la déchirure de 
Nomenclature

\begin{tabular}{|c|c|}
\hline$C_{r k}$ & couple de frottements arbre-rouleau du $k^{\text {ème }}$ \\
\hline & rouleau, \\
\hline$J_{k}$ & inertie du $k^{\text {ème }}$ rouleau \\
\hline$K_{k} U_{k}$ & couple moteur (si le $k^{\text {ème }}$ rouleau est motorisé) \\
\hline$L_{k}$ & $\begin{array}{l}\text { longueur de bande entre le } k^{\text {ème }} \text { et le }(k+1)^{\text {ème }} \\
\text { rouleaux }\end{array}$ \\
\hline$R_{k}$ & rayon du $k^{\text {ème }}$ rouleau \\
\hline$T_{k}$ & $\begin{array}{l}\text { tension de bande entre le } k^{\text {ème }} \text { et le }(k+1)^{\text {ème }} \\
\text { rouleaux }\end{array}$ \\
\hline$V_{k}$ & vitesse linéaire du $k^{\text {ème }}$ rouleau \\
\hline$\varepsilon_{k}$ & $\begin{array}{l}\text { allongement du brin de bande entre le } k^{\text {ème }} \\
\text { et le }(k+1)^{\text {ème }} \text { rouleaux }\end{array}$ \\
\hline$\Omega_{k}$ & vitesse angulaire du $k^{\text {ème }}$ rouleau \\
\hline
\end{tabular}

la bande. Parmi les nombreux incidents on peut citer les plis de bande, le déport latéral pendant le transport, une surtension de bande.

Une ligne industrielle d'entraînement de bandes flexibles est constituée de plusieurs sous-systèmes. Généralement le dérouleur et l'enrouleur sont disposés respectivement au début et à la fin de la ligne. Les autres éléments, tels que les rouleaux danseurs, les moteurs tracteurs, les rouleaux libres ou les accumulateurs, sont utilisés et disposés en fonction du procédé de traitement employé. Un exemple est donné à la figure 1. Différents éléments de traitement sont inclus dans une ligne de production, comme par exemple l'impression, l'enduction ou le séchage.

Le danseur représente un cas particulier du rouleau libre : il a la possibilité supplémentaire de se mouvoir. Il possède une double utilisation. D'une part, il impose la tension de bande au dérouleur ou à l'enrouleur près duquel il est placé. D'autre part, il sert de filtre mécanique afin d'atténuer, et dans certains cas même de supprimer, les variations rapides de tension provoquées notamment par les défauts géométriques d'une bobine.

L'accumulateur trouve son utilisation dans les systèmes industriels en défilement continu de la bande. Il stocke la bande pendant le fonctionnement normal de la ligne et la restitue pendant le changement de bobine. Durant la phase d'accumulation, la partie supérieure de l'accumulateur vertical est déplacée vers le haut, ce qui impose inévitablement une vitesse de bande à son entrée supérieure à celle de la sortie. Pendant la phase de déstockage cependant, le moteur d'entrée est à l'arrêt et la partie supérieure de l'accumulateur se rapproche de la partie inférieure de manière à garder constante la vitesse de la bande à sa sortie. Durant cette phase relativement courte, la bobine de déroulement vide est remplacée par une bobine pleine. L'accumulateur peut être à déplacement horizontal ou vertical. Cependant pour les lignes à vitesse de défilement élevée et qui nécessitent par conséquent une grande capacité de stockage, l'accumulateur vertical est privilégié.

La présente étude se focalise sur le comportement dynamique de systèmes d'entraînement de bandes flexibles en fonction de l'élasticité de la bande.

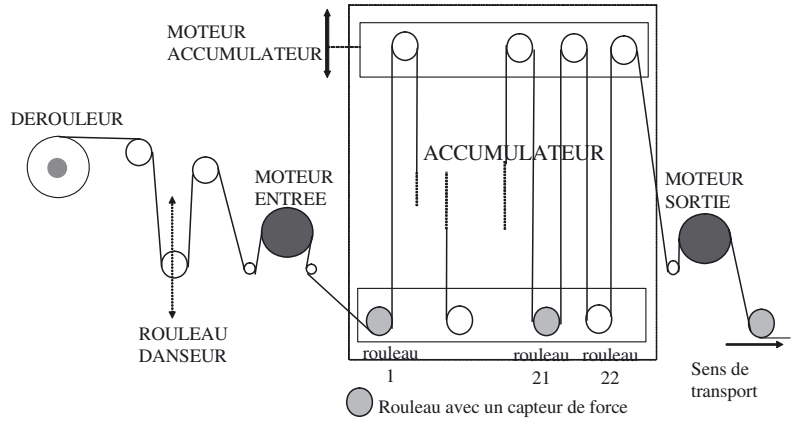

Fig. 1. Principe d'un système industriel avec accumulateur (côté dérouleur).

\section{Modélisation du système}

Les systèmes multi-moteurs d'entraînement de bandes flexibles sont des systèmes à grande échelle, multivariables, non-linéaires et à paramètres variant dans le temps et constituent à ce titre des systèmes complexes. Ils sont composés de nombreux éléments qui assurent chacun une ou plusieurs fonctions bien définies. Le couplage entre eux est réalisé par la bande elle-même. L'étude du comportement dynamique du système nécessite l'élaboration d'un modèle de connaissance. À l'inverse d'un modèle comportemental de type boîte noire, une modélisation phénoménologique permet de connaître l'influence des variations des paramètres.

La vitesse de bande et sa tension sont des grandeurs primordiales dans tout système de production industrielle. Elles sont obtenues par l'action des rouleaux motorisés sur la bande.

\subsection{Vitesse de bande}

La vitesse du $k^{\text {ème }}$ rouleau est déterminée à partir du théorème du moment dynamique appliqué à ce dernier. En supposant l'absence de glissement entre la bande et le rouleau, la vitesse $V_{k}$ de la bande est égale à la vitesse périphérique du rouleau. La relation s'exprime [1,2] :

$$
\frac{\mathrm{d}\left(J_{k} \Omega_{k}\right)}{\mathrm{d} t}=R_{k}\left(T_{k}-T_{k-1}\right)+K_{k} U_{k}-C_{r k}
$$

\subsection{Tension de bande}

Le calcul de la tension de bande entre deux rouleaux consécutifs est basé sur l'équation de continuité $[1,2]$ :

$$
\frac{\mathrm{d}}{\mathrm{d} t}\left(\frac{L_{k}}{1+\varepsilon_{k}}\right)=-\frac{V_{k+1}}{1+\varepsilon_{k}}+\frac{V_{k}}{1+\varepsilon_{k-1}}
$$

La loi de Hooke (en supposant un comportement élastique) permet de déduire l'équation de la tension de bande $[1,2]$ :

$$
\begin{aligned}
& L \frac{\mathrm{d} T_{k+1}}{\mathrm{~d} t}= \\
& \quad E S\left(V_{k+1}-V_{k}\right)+T_{k} V_{k}-T_{k+1}\left(2 V_{k}-V_{k+1}\right)
\end{aligned}
$$




\subsection{Modélisation des éléments constituant une ligne d'entraînement de bande}

Le modèle complet d'un système d'entraînement de bandes est construit par l'assemblage des modèles des éléments primitifs (dérouleur, danseur, accumulateur, rouleaux motorisés ou libres, ...). Une récurrence est ensuite établie entre les tensions de bande et les vitesses des rouleaux des éléments primitifs voisins. La modélisation de chaque élément primitif est basée sur les équations (1) et (2), et sur le modèle rhéologique de la bande. Des modélisations sont données dans [1-5].

\section{Influence des propriétés surfaciques de la bande}

Des essais ont été réalisés sur un banc à 3 moteurs (dérouleur, tracteur, enrouleur), la rugosité du rouleau tracteur est comparable à celle utilisée dans l'industrie.

$\mathrm{Si}$ les variations de consignes de vitesse sont imposées facilement au tracteur, celles de l'enrouleur et du dérouleur durant les phases transitoires sont tributaires des glissements qui apparaissent dans le contact bande/rouleau tracteur. Lors d'une augmentation de vitesse, habituellement la méthode employée est la suivante : la vitesse est augmentée régulièrement pendant que la tension de la bande est maintenue constante. Cependant, des expérimentations ont montré qu'une augmentation en échelon de la consigne de tension conjointement à la vitesse permettait d'atteindre plus rapidement la phase d'adhérence et donc la consigne de vitesse. L'adhérence bande-rouleau dépend de la force de frottement entre la bande et le rouleau donc de la tension de la bande, dont dépend la force normale au rouleau, mais également de la rugosité du rouleau tracteur par rapport à la bande et de leur adhésion.

La figure 2 montre d'une part la consigne en fonction du temps, et d'autre part la vitesse linéaire et la variation de la pente lorsque l'échelon en tension est appliqué. L'apport de cette méthode est quantifié à partir du temps nécessaire pour atteindre la consigne $(2 \mathrm{~s}$ pour atteindre la consigne après un saut de vitesse, à comparer aux $5 \mathrm{~s}$ après une rampe de vitesse sans saut de tension). L'amélioration obtenue n'a pas été atteinte par la synthèse de commandes élaborées [2].

\section{Influence des propriétés volumiques de la bande : influence de l'élasticité}

Le comportement en traction de la bande varie en fonction des conditions de production ou de transformation. En effet, l'humidité et la température sont deux facteurs prépondérants. De plus, pour certains types de matériaux tels que les textiles, les caractéristiques rhéologiques changent en fonction des conditions de sollicitation, à savoir la tension appliquée et la vitesse d'application. À titre d'exemple, la figure 3 met en évidence vitesses linéaires $(\mathrm{m} / \mathrm{m}$ in $)$

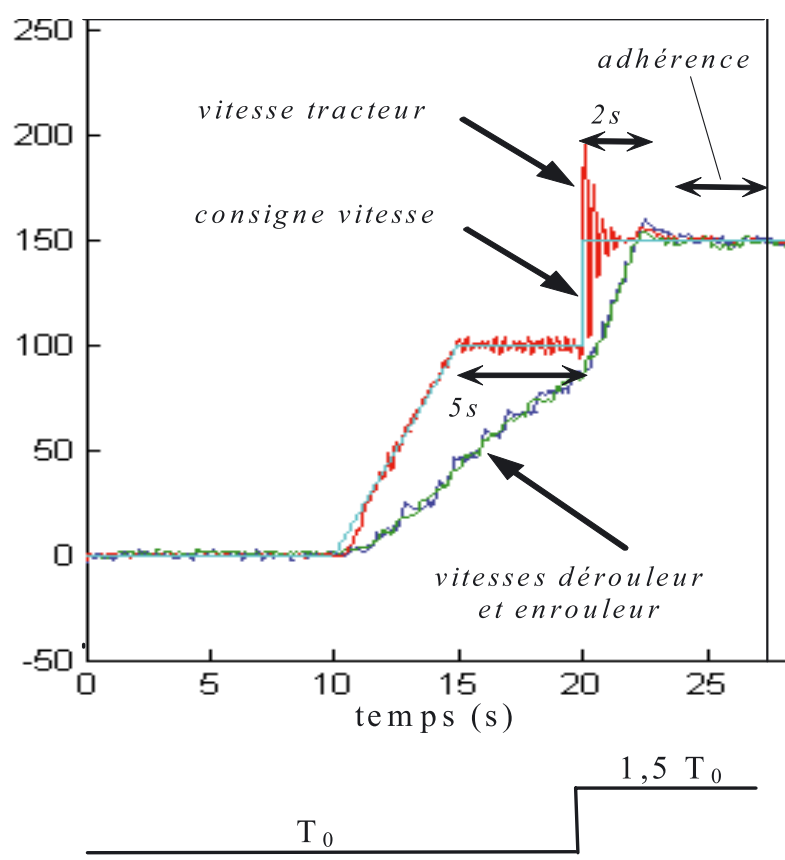

Evolution de la consigne de tension

Fig. 2. Mesures des vitesses linéaires de la bande relevées sur un banc 3 moteurs. La tension de bande est maintenue constante jusqu'à $t=20 \mathrm{~s}$. À cet instant, l'augmentation de la consigne de tension est effectuée conjointement avec celle de la vitesse. Les différences de vitesses linéaires entre dérouleur (et enrouleur) et tracteur correspondent au patinage bande/tracteur.

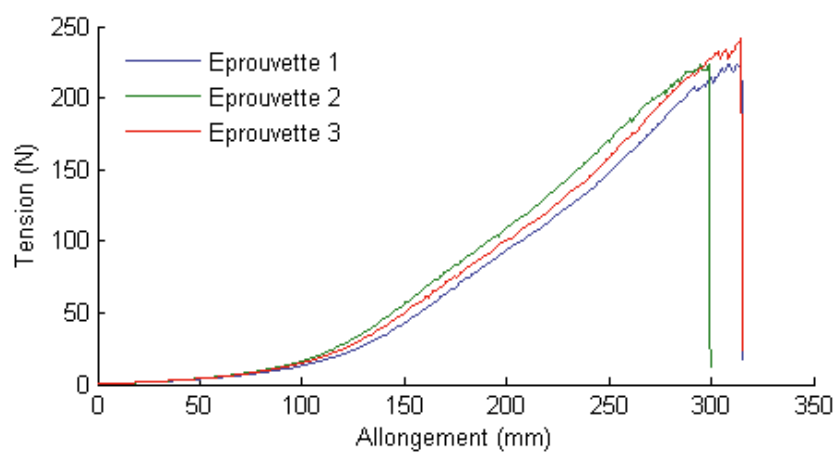

Fig. 3. Courbe force-allongement d'un tricot.

les variations de la pente de la courbe force-allongement dans le cas d'un tricot.

Ces caractéristiques non linéaires, nécessaires dans la synthèse de correcteurs pour la commande des machines, peuvent être déterminées « hors ligne» à l'aide d'une machine de traction ou « en ligne » à l'aide d'un extensomètre optique [6]. Dans cet extensomètre, un trait laser est projeté sur la bande perpendiculairement à son sens de défilement et l'énergie des rayons réfléchis est récupérée par une photodiode. L'étude fréquentielle du signal réfléchi permet de déduire la variation du nombre 


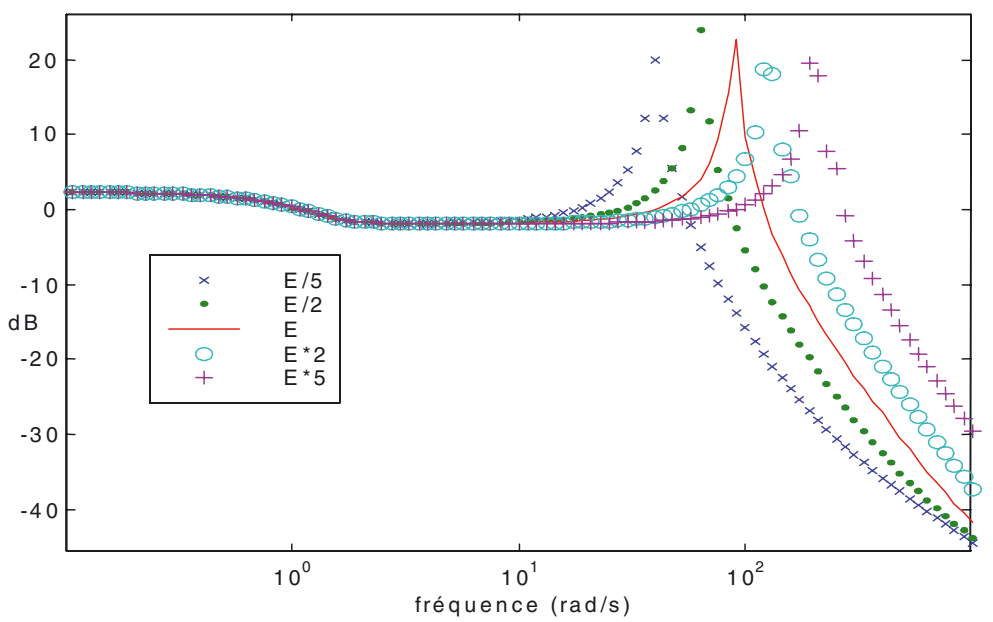

Fig. 4. Comportement fréquentiel (valeur singulière maximale) d'un système à 3 moteurs en fonction du module de Young.
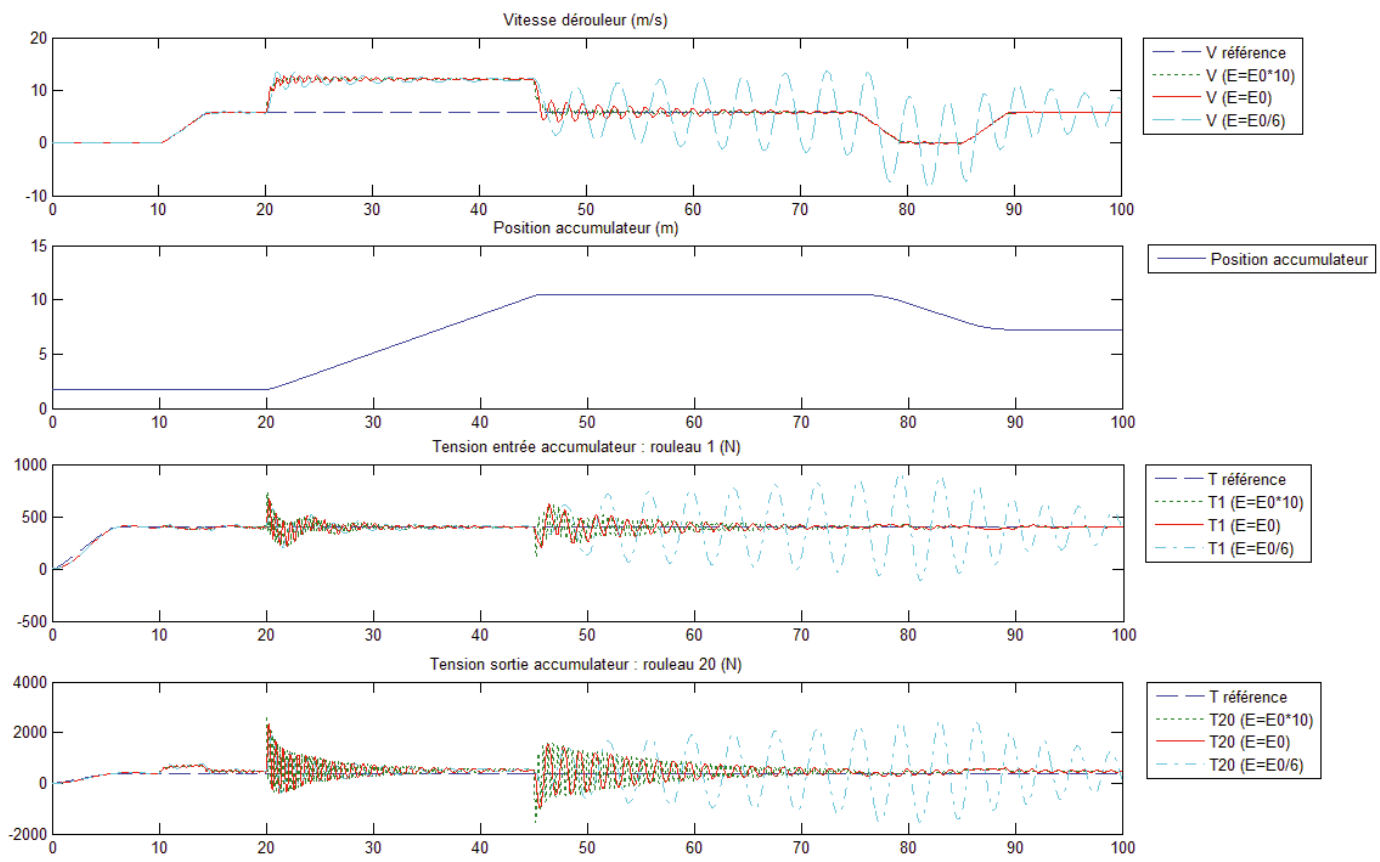

Fig. 5. Vitesse et tensions de bande (pour différents modules de Young) d'un sous-système composé d'un dérouleur et d'un accumulateur : la commande devient instable lorsque le module de Young nominal est divisé par 6.

d'éléments du relief (trame par exemple) par unité de temps et ainsi de déterminer l'élongation en fonction de la vitesse de défilement et de la tension mesurée. L'exploitation de cette mesure d'élongation couplée à une information de tension disponible dans la même zone du système permet d'aboutir à une estimation de l'élasticité du matériau de la bande. Cette valeur ainsi obtenue peut être utilisée comme variable d'ajustement d'une commande adaptative.

\section{1 Étude fréquentielle du système}

La robustesse de la commande vis-à-vis d'une variation de l'élasticité est un critère important pour le choix définitif du correcteur pour un système donné. Cette robustesse a été analysée dans [2,9].

La figure 4 montre que, la fréquence de résonance du système à 3 moteurs (composé d'un dérouleur, d'un tracteur et d'un enrouleur) en boucle ouverte, c'est-à-dire non asservi par des correcteurs, dépend du module de Young. Si cette fréquence de résonance du système entre dans la bande passante du système bouclé (c'est-à-dire corrigé avec des correcteurs), les performances du système asservi peuvent être dégradées. Dans le cas extrême, le système bouclé peut devenir instable si auparavant la synthèse de la commande n'a pas tenu compte des variations du module de Young. Un exemple est donné dans la figure 5 pour le cas d'un sous-système composé d'un dérouleur et d'un accumulateur. 
Le choix d'une structure de commande et la synthèse des correcteurs associés doivent par conséquent tenir compte, entre autres, des variations des propriétés élastiques de la bande. Des exemples de commande robustes $\mathrm{H}_{\infty}$ (LTI, LPV, multi-modèles, ...) sont donnés dans $[1,2,5,7,8,10]$.

\section{Conclusions}

Les propriétés surfaciques et volumiques ont une grande importance dans l'optimisation des performances d'un système de déroulement-enroulement et d'entraînement de bandes flexibles. Il est important de quantifier leurs influences afin d'en tenir compte dans le choix de la structure et de la synthèse de commandes robustes. Certains paramètres comme l'élasticité de la bande peuvent être déterminés en ligne afin de les utiliser comme paramètres d'ajustement pour une commande adaptative.

\section{Références}

[1] D. Knittel, M. Vedrines, D. Henrion, P.R. Pagilla, Robust $\mathrm{H}_{\infty}$ fixed order control strategies for large scale web winding systems, IEEE Conference on Control Applications CCA/CACSD/ISIC, Germany, October 4-6, 2006

[2] H. Koc, D. Knittel, M. De Mathelin, et G. Abba, Modeling and robust control of winding systems for elastic webs, IEEE Transactions on Control Systems Technology 10 (2002) 197-206
[3] W. Wolfermann, Tension control of webs, a review of the problems and solutions in the present and future, 3rd International Conference on Web Handling, Stillwater, OK (USA), 1995, pp. 198-229

[4] N.A. Ebler, R. Arnason, G. Michaelis, et N. D'Sa, Tension control: dancer rolls or load cells, IEEE Transactions on Industry Applications 29 (1993) 727-739

[5] M. Vedrines, D. Knittel , D. Guy, E. Caillaud, Modeling and control of an industrial winder with pendulum dancer mechanism, 4th IFAC-Symposium on Mechatronic Systems Heidelberg, IFAC-ASME, Germany, September 12-14, 2006

[6] M. Tourlonias, M.-A. Bueno, L. Bigué, B. Durand, M. Renner, Contactless optical extensometer for textile materials, Experimental Mechanics 45 (2005) 420-426

[7] A. Benlatreche, D. Knittel, E. Ostertag, Robust decentralized control strategies for large scale web handling systems, IFAC J. Control Engineering Practice 16 (2008) $736-750$

[8] D. Knittel, E. Laroche, D. Gigan, H. Koç, Tension control for winding systems with two degrees of freedom $\mathrm{H}_{\infty}$ controller, IEEE Transactions on Industry Applications 39 (2003) 113-120

[9] D. Knittel, Robust control design using $\mathrm{H}_{\infty}$ methods in large scale web handling systems, 7th Int. Conf. on Web Handling, IWEB2003, Stillwater, Oklahoma, 2003

[10] D. Knittel, A. Arbogast, M. Vedrines, P. Pagilla, Decentralized robust control strategies with model based feedforward for elastic web winding systems, American Control Conference, Minnesota, 2006

[11] V. Gassmann, D. Knittel, $\mathrm{H}_{\infty}$ - based PI-observers for web tension estimations in industrial unwinding-winding systems, IFAC World Congress, Seoul, 2008 